\title{
Transcutaneous electrical nerve stimulation (TENS) for primary dysmenorrhea: a systematic review and meta-analysis
}

\author{
Kyoung Sun Park' ${ }^{1}$ Sola Han², Hae Sun Suh², Jin Moo Lee ${ }^{1}$ \\ 1 Department of Korean Medicine Obstetrics \& Gynecology, College of Korean Medicine, Kyung Hee \\ University, South Korea \\ ${ }^{2}$ College of Pharmacy, Pusan National University, South Korea
}

\section{Problem statement}

Primary dysmenorrhea is defined as cramping pain during menstruation without any identifiable pelvic pathology, leading to the restriction of daily activities. Nonsteroidal anti-inflammatory drugs (NSAIDs) and oral contraceptives (OCs) are recommended as a first-line treatment. Transcutaneous electrical nerve stimulation (TENS) is a non-pharmacological intervention shown to be effective for pain relief in a variety of conditions. Our study aim to determine the efficacy of high- and low-frequency TENS compared to placebo for primary dysmenorrhea.

\section{Methods}

We searched the following 16 electronic databases for relevant articles published before November 2016: MEDLINE, EMBASE, CENTRAL, Allied and complementary medicine database (AMED), Cumulative Index to Nursing and Allied Health Literature (CINAHL), KoreaMed, Korean studies Information Service System (KISS), Korean medical database (KMbase), National Digital Science Library (NDSL), Korean Institute of Science and Technologe Information (KISTI), Korean Traditional Knowledge Portal, Oriental Medicine Advanced Searching Integrated System (OASIS), China National Knowledge Infrastructure (CNKI), Wanfang, VIP, and CiNii. The inclusion criteria were RCTs of TENS compared to placebo, no treatment, or medical treatment for primary dysmenorrhea of reproductive age.
Exclusion criteria were secondary dysmenorrhea due to any pelvic pathology. Risk of bias in each article was assessed according to Cochrane risk of bias tool for RCTs by three independent reviewer. For the statistical analysis, the mean differences (MD) or standardized mean differences (SMD) was calculated with $95 \%$ confidence intervals (Cls) using the Review Manager software (RevMan v5.3).

\section{Results}

80 RCTs were finally included in systematic review, and metaanalysis was done with 16 RCTs. Most studies showed low or unclear risk of biases. High-frequency TENS was shown to be more effective than placebo TENS (SMD -1.84, 95\% Cl [-3.16, 0.51], $P=0.007$, Fig. 1(A)). Low-frequency TENS was found to be no more effective in reducing pain compared to placebo TENS (SMD -2.80, 95\% Cl [-5.67, 0.07], $\mathrm{P}=0.06$, Fig. 1(B)). It is still uncertain that high-frequency TENS was more effective than low-frequency TENS (SMD 1.47, 95\% Cl [-0.17, 3.11], $\mathrm{P}=0.08$, Fig. 1(C)).

\section{Conclusion}

The result of this study shows that high-frequency TENS was shown to be more effective than placebo TENS, whereas lowfrequency TENS was found to be no more effective in reducing dysmenorrhea compared to placebo TENS. Additional studies to convince that high-frequency TENS is more effective than lowfrequency TENS are needed.
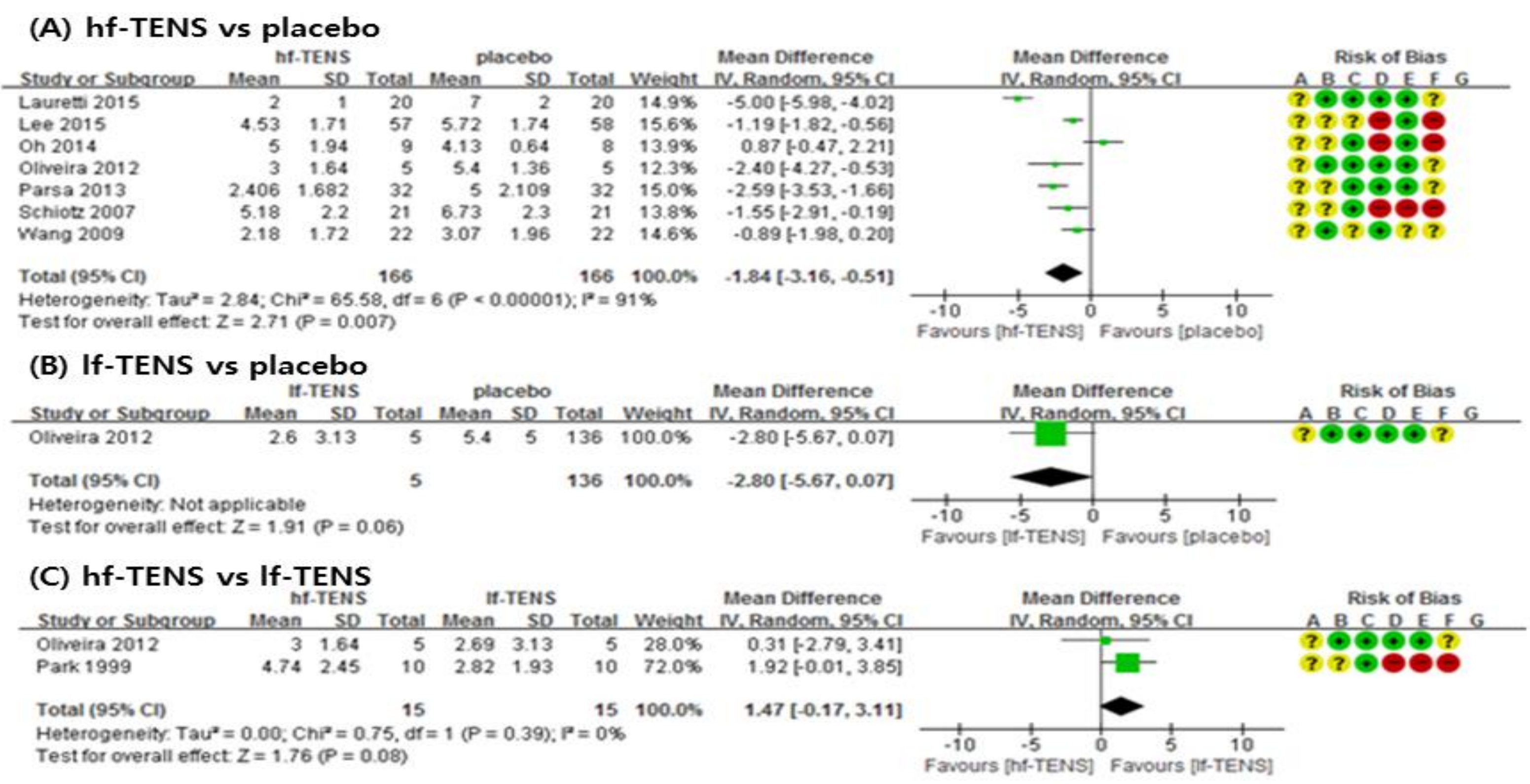